\title{
Thin Light Tube Formation by Tightly Focused Azimuthally Polarized Light Beams
}

\author{
Svetlana N. Khonina and Andrey V. Ustinov \\ Image Processing Systems Institute of the Russian Academy of Sciences, Molodogvardeiskaya Street 151, Samara 443001, Russia \\ Correspondence should be addressed to Andrey V. Ustinov; andr@smr.ru
}

Received 21 June 2013; Accepted 14 July 2013

Academic Editors: A. K. Dharmadhikari and D. Poitras

Copyright (c) 2013 S. N. Khonina and A. V. Ustinov. This is an open access article distributed under the Creative Commons Attribution License, which permits unrestricted use, distribution, and reproduction in any medium, provided the original work is properly cited.

\begin{abstract}
Theoretical and numerical analysis of the transmission function of the focusing system with high numerical aperture was conducted. The purpose of the study was to form a thin light tube in a focal area using the azimuthally polarized radiation. It was analytically shown that, due to destructive interference of two beams formed by two narrow rings, it is possible to overcome not only the full aperture diffraction limit but also the circular aperture limit. In this case, however, the intensity at the center of the focal plane is significantly reduced, which practically leads to the tube rupture. It was numerically shown that long thin one-piece tubes may be formed through the aperture apodization with diffractive axicon phase function or with complex transmission function of Laguerre-Gaussian or Airy-Gaussian beams.
\end{abstract}

\section{Introduction}

Introducing a narrow annular aperture to the tightly focused cylindrical beams with radial or azimuthal polarization, the blocking light in almost all central parts of the lens [13 ] is a simple but energetically expensive way of forming long narrow beams in the focal region. In the case of radial polarization a thin thread of light is formed, while in the azimuthal polarization a light tube is formed. Moreover, the transverse dimension corresponds to the scalar diffraction limit. In other types of polarization the focal spot (or ring) is larger because of the contribution of the various components of the electromagnetic field to the focal region.

To increase the efficiency and overcome the diffraction limit more sophisticated ways of full aperture apodization of function are used. It can be either a pure phase or an amplitude-phase distribution [4-9]. Thus, as a rule, reducing the focal spot size is accompanied by the redistribution of energy from the central part to the sidelobes. This situation is consistent with the Toraldo di Francia theory [10], according to which possible to obtain infinitely narrow central spot due to the growth of sidelobes. But this growth is sometimes several times $[8,9]$ or even orders [11] higher than the reducing of central light spot.

The presence of significant sidelobes limits the use of "super-resolution" elements in representing systems and optical data recording, when the acceptable intensity level in the sidelobes is less than $30 \%$ with respect to the central peak [12].

However, the optimization procedures controlling the growth of the sidelobes lead to the inevitable broadening of the central spot size $[12,13]$.

It was shown in [14] that the introduction of the radial phase jump on $\pi$ radians for the midradius of the narrow annular aperture leads to a reduction of the central peak size to $\mathrm{FWHM}=0.33 \lambda$ for the radial polarization, which is less than a simple circular aperture result $(\mathrm{FWHM}=0.36 \lambda)$. In this case, the intensity level of the sidelobes is less than $30 \%$ of the main peak, and they can be filtered out [15] or neutralized by the nonlinear interaction of light and the record medium [16].

Reducing the focal spot size without a significant increase of sidelobes is achieved by introduction of the radial phase jump due to destructive interference of two beams, formed by each of the rings in the aperture $[14,17]$. 
Theoretical and numerical analysis of the transmission function of the focusing system with high numerical aperture was conducted in this paper in order to form a thin light tube in the focal area using azimuthal polarization. This distribution is used in optical trapping and manipulation of solid microparticles and cold atoms [18, 19], in shadow microscopy, as well as in microscopy based on stimulated emission depletion: STED [20, 21].

\section{Theoretical Analysis}

Azimuthally polarized vector electric field of high-aperture focusing optical system in a homogeneous dielectric medium near the focus can be described by the following expression:

$$
\begin{aligned}
\mathbf{E}(\rho, \varphi, z)= & \left(\begin{array}{c}
E_{r}(\rho, \varphi, z) \\
E_{\varphi}(\rho, \varphi, z) \\
E_{z}(\rho, \varphi, z)
\end{array}\right) \\
= & k f \int_{0}^{\Theta} D(\theta)\left[\begin{array}{c}
0 \\
J_{1}(k \rho \sin \theta) \\
0
\end{array}\right] \\
& \quad \times \sqrt{\cos \theta} \sin \theta \exp (i k z \cos \theta) \mathrm{d} \theta,
\end{aligned}
$$

where $f$ is focal distance, $k=2 \pi / \lambda$ is wave number, $\sin \Theta=$ $\mathrm{NA} / n, \mathrm{NA}$ is numerical aperture of lens, $n$ is refractive index of the medium, $J_{1}(\cdot)$ is Bessel function of the first kind and the first order, and $D(\theta)$ is any univariate function.

It can be seen from expression (1) that the light ring is formed in the focal plane in scalar mode; all the components except the azimuth one are zero. This fact makes it easy to implement various optimization procedures to correct distribution in the focal plane, in particular to reduce the size of the light ring.

The introduction of a narrow annular aperture that transmits light only in the peripheral part of the lens can reduce the inner ring to the scalar limit that corresponds to the first-order Bessel function:

$$
\begin{aligned}
I_{\delta}(\rho, \varphi, 0) & =\int_{\Theta-\delta}^{\Theta} \sin \theta \sqrt{\cos \theta} J_{1}(k \rho \sin \theta) \mathrm{d} \theta \\
& \approx \int_{1-\Delta}^{1} x\left(1-x^{2}\right)^{-1 / 4} J_{1}(k \rho x) \mathrm{d} x \\
& \approx \frac{2}{3} \Delta^{3 / 4}(2-\Delta)^{3 / 4} J_{1}\left(k \rho\left(1-\frac{\Delta}{2}\right)\right) .
\end{aligned}
$$

The focal distribution along the optical axis elongates simultaneously. A thin light tube is a result of this process. The radius of such tube is determined by the maximum value of the Bessel function:

$$
\rho_{0}=\frac{1.84 \lambda}{2 \pi}\left(1-\frac{\Delta}{2}\right)^{-1} \underset{\Delta \rightarrow 0}{\longrightarrow} 0.293 \lambda
$$

and approximately equals the full width at half-maximum (FWHM).
Note that the narrowest transverse dimension of the light tube is achieved by annular aperture width tends to zero. In this case, the intensity of expression (2) also tends to zero. This fact is the main disadvantage of such approach.

Also there are some full-aperture distributions that allow more efficient forming of thin light tubes in azimuthal polarization-Zernike polynomials [9], axicon-like structures [8], and Laguerre-Gaussian (LG) modes [22, 23], as well as binary phase elements $[24,25]$.

To reduce the diameter of the light tube the optimization [13] of function $D(\theta)$ can be performed, but in this case a good length of the tube is not guaranteed. To comply with both conditions it is easier to combine the optimization procedure with the central locking of the lens.

We should also keep in mind the complexity of the manufacturing of optical elements for apodization pupil of the focusing system. A binary structure is the most suitable for it, that is, when the function $D(\theta)$ has a ring structure with two constant values $c_{p}= \pm 1, \theta_{p-1} \leq \theta \leq \theta_{p}$.

The following function can be used to decrease the focal ring radius:

$$
D(\theta)= \begin{cases}0, & 0 \leq \theta<\Theta-2 \delta \\ 1, & \Theta-2 \delta \leq \theta<\Theta-\delta \\ -1, & \Theta-\delta \leq \theta \leq \Theta\end{cases}
$$

Function (4) allows us to achieve the beams interference formed by separate rings in the focus (2):

$$
\begin{aligned}
I_{\tau}(\rho, \varphi, 0)= & \int_{\Theta-\delta}^{\Theta} \sin \theta \sqrt{\cos \theta} J_{1}(k \rho \sin \theta) \mathrm{d} \theta \\
& -\int_{\Theta-2 \delta}^{\Theta-\delta} \sin \theta \sqrt{\cos \theta} J_{1}(k \rho \sin \theta) \mathrm{d} \theta \\
\approx & \int_{1-\Delta}^{1} x\left(1-x^{2}\right)^{-1 / 4} J_{1}(k \rho x) \mathrm{d} x \\
& -\int_{1-2 \Delta}^{1-\Delta} x\left(1-x^{2}\right)^{-1 / 4} J_{1}(k \rho x) \mathrm{d} x \\
= & \frac{2}{3} \Delta^{3 / 4}\left[(2-\Delta)^{3 / 4} J_{1}\left(k \rho\left(1-\frac{\Delta}{2}\right)\right)\right. \\
& -\left[(4-4 \Delta)^{3 / 4}-(2-\Delta)^{3 / 4}\right] \\
& \left.\times J_{1}\left(k \rho\left(1-\frac{3 \Delta}{2}\right)\right)\right] .
\end{aligned}
$$

Using the approximation of Bessel function [26],

$$
J_{1}(x) \approx \frac{x}{2}-\frac{x^{3}}{16}
$$


$D(\theta)$, amplitude and phase

Ring, $\Delta=0.05$
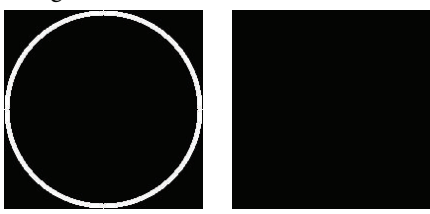

Mode LG (1,1), $\sigma=35 \lambda$
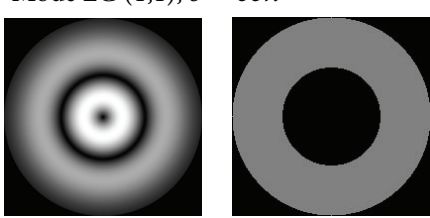

Optimized
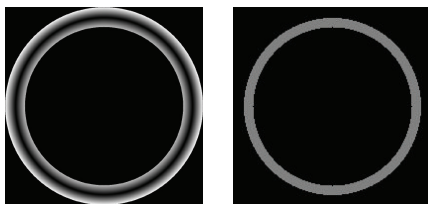

Ring with a jump, $\Delta=0,1$
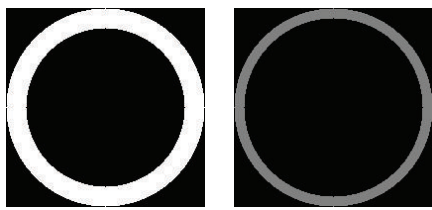

Transverse intensity in the focus $4 \lambda \times 4 \lambda$
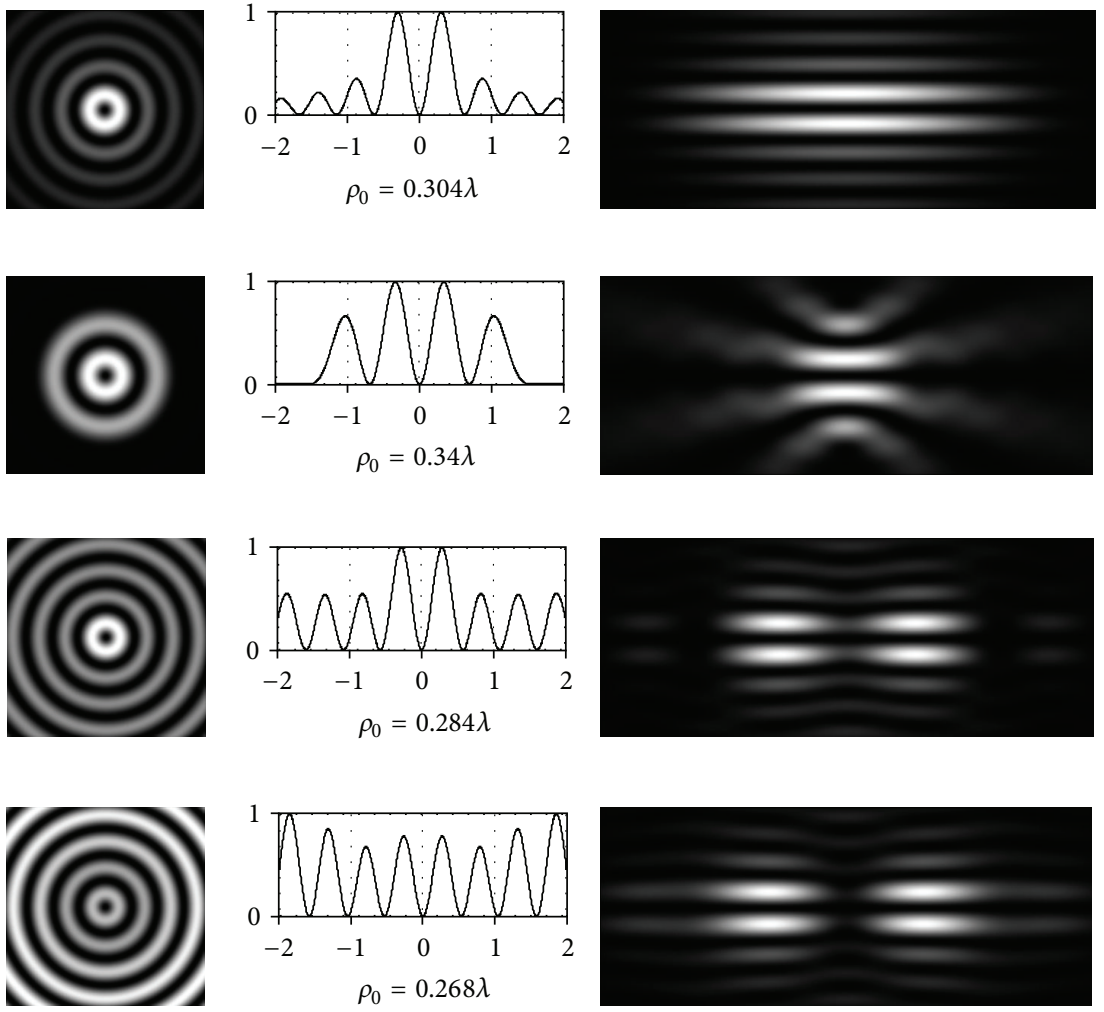

Longitudinal intensity $10 \lambda \times 4 \lambda$
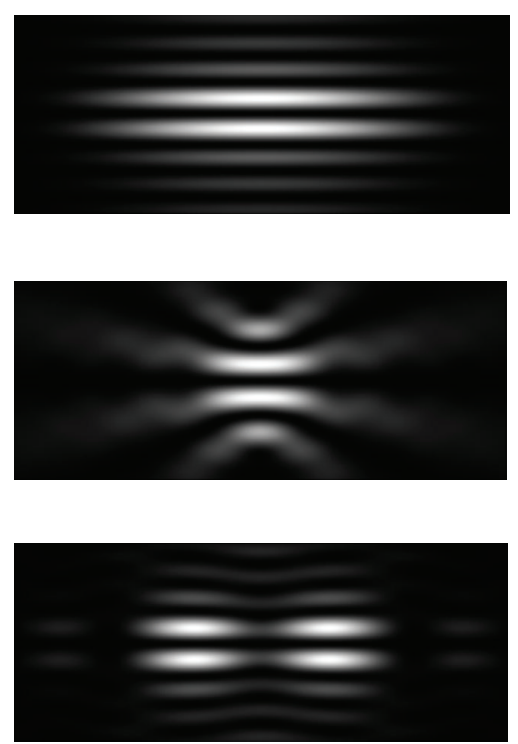

FIGURE 1: Thin light tube formation.

The following approximation can be obtained from expression (5):

$$
\begin{aligned}
I_{\tau}(\rho, \varphi, 0) & \\
\approx \frac{2}{3}(2 \Delta)^{3 / 4} & {\left[\frac{k \rho}{2}\left(0.318+1.034 \Delta-1.14 \Delta^{2}\right)\right.} \\
& \left.-\frac{(k \rho)^{3}}{16}\left(0.318+2.08 \Delta-7.167 \Delta^{2}\right)\right],
\end{aligned}
$$

The value $\Delta$ providing the minimum light ring size in the focal plane can be found analytically. The maximum of expression (7) is achieved when

$$
\left(k \rho_{\tau}\right)^{2}=\frac{8}{3} \frac{\left(0.318+1.034 \Delta-1.14 \Delta^{2}\right)}{\left(0.318+2.08 \Delta-7.167 \Delta^{2}\right)} .
$$

It can be seen from expression (8) that the minimum focal ring radius is provided by $\Delta_{\min } \approx 0.0788$. In this case,

$$
\rho_{\tau, \min } \approx 0.246 \lambda \text {. }
$$

Because of the Bessel function (6) approximation the estimate (9) is understated by about $6 \%$, that is, $\rho_{\tau, \text { min }} \approx$ $0.26 \lambda$. But even in this case the corresponding scalar limit is overcome.

\section{Numerical Simulation}

The results of numerical simulation for the expression (1) for the different transmission functions $D(\theta)$ are listed in this section. Focal length was chosen to be equal to $f=101 \lambda$, the numerical aperture of the lens NA $=0.99$. The focusing was considered in the air $n=1$, so that the maximum azimuthal angle in (1) is $\Theta \approx 82^{\circ}$. This provides sufficient accuracy of (2) and (5).

The results of the simulation for a narrow annular aperture width $\Delta=0.05$ (first line) are listed in Figure 1. In this case according to approximation (3) the radius of the light ring $\rho_{0}=0.3 \lambda$ is very close to the numerical results $\rho_{0}=0.304 \lambda$.

To increase efficiency, you can use modes of laser radiation. The results for the mode LG $(1,1)$ are shown in the second row of Figure 1. However in this case the radius of the light tube increases $\rho_{0}=0.34 \lambda$. The structure of the illuminating field is practically repeated in the focal plane. It has the appropriate number of rings. With the increase of the radial mode number the efficiency in the tube center falls and the diameter reduces.

Significant reduction of the tube diameter can be achieved through an iterative calculation [13]. The results of this calculation with the controlled growth of sidelobes are shown in the third row of Figure 1. In this case the diffraction limit ( $\rho_{0}=0.284 \lambda$ ) was overcome, but the light tube has a lower intensity in the center part. 
$D(\theta)$, amplitude and phase

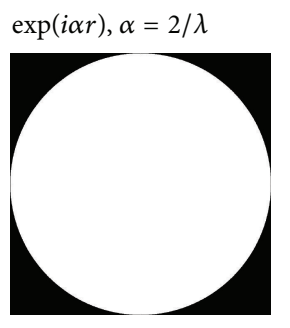

$\cos (\alpha r), \alpha=1 / \lambda$

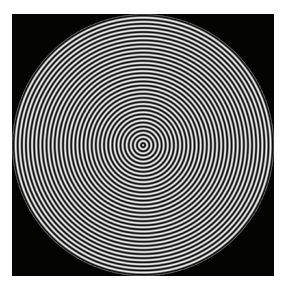

Mode LG $(18,1), \sigma=10 \lambda$

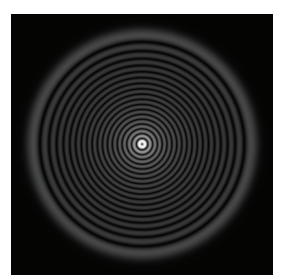

Function AG, $n=21, \sigma=60 \lambda$

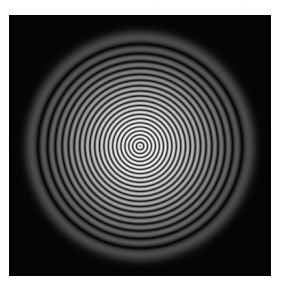

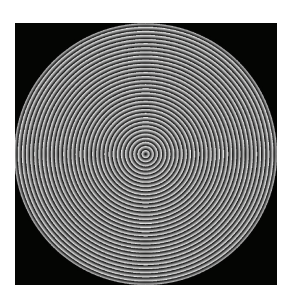

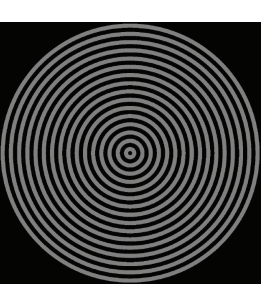

$z \in[-30 \lambda, 30 \lambda], r \in[0,2 \lambda]$

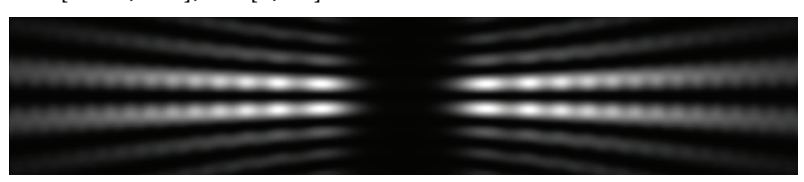

$\rho_{0}(z=5 \lambda)=0.304 \lambda ; \rho_{0}(z=30 \lambda)=0.65 \lambda$

$z \in[-60 \lambda, 60 \lambda], r \in[0,2 \lambda]$

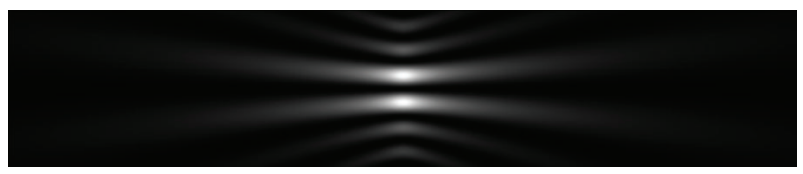

$\rho_{0}(z=0)=0.34 \lambda ; \rho_{0}(z=60 \lambda)=1.33 \lambda$

$z \in[-60 \lambda, 60 \lambda], r \in[0,2 \lambda]$

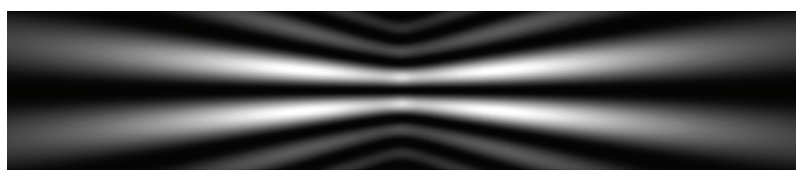

$\rho_{0}(z=0)=0.336 \lambda ; \rho_{0}(z=60 \lambda)=1.2 \lambda$

Figure 2: Long light tube formation.

Zernike polynomial of the second order allows you to create a similar light tube [9], its radius $\rho_{0}=0.28 \lambda$, but the diameter reduction is achieved by increasing the sidelobes.

Approach (4) allows further tube diameter reduce in the focal plane $\left(\rho_{0}=0.268 \lambda\right)$, but the intensity also further reduces. In this case, the picture in the focal area looks almost like a tube with a gap.

To get very long light tubes diffractive axicon can be used as a transmission phase function $\exp (i \alpha r)$. This system works as high-aperture lensacon [27]. The first line of Figure 2 shows the formation of a narrow cone, which begins a little further from focal plane and extends to a distance of more than 60 wavelengths. The radius of the cone is small: in the beginning (at a distance $z=5 \lambda) \rho_{0}(z=5 \lambda)=0.3 \lambda$ and in the end (at a distance $z=60 \lambda) \rho_{0}(z=60 \lambda)=0.63 \lambda$.

If the superposition of two axicons-divergence and convergence is used: $\exp (i \alpha r)+\exp (-i \alpha r)=2 \cos (\alpha r)$, it is possible to form two symmetrical cones at the same time (see the second line of Figure 2). However, in this case there is an open loop of right and left light beams.
As it was mentioned earlier, LG modes of high order can be used to form thin solid light tubes. In the third row of Figure 2 the result for the mode of LG $(18,1)$ is shown. In this case, a long one-piece light tube is formed, its radius in the center of tube is $\rho_{0}(z=0)=0.34 \lambda$ and at a distance of 60 wavelengths is about 4 times more: $\rho_{0}(z=60 \lambda)=$ $1.33 \lambda$. Thus, the divergence of such tube is greater than that for the axicon and corresponds to a Gaussian beam. Due to the high divergence the intensity of tube decreases rapidly with increasing distance from the focal plane. Longitudinal intensity of the tube can be made more uniform if the Gaussian parameter $\sigma$ is reduced. In this case illuminating radiation is concentrated in the central part of the lens, the numerical aperture is reduced, and the divergence of the Gaussian beam is reduced by the focal radius increasing.

Another result is obtained if the transmission function is a radial Airy-Gaussian function (AG). This distribution was considered in [28] as close to Gaussian modes, and it was shown that more compact light beam is formed in the focal plane. Indeed, the light tube is a little more narrow: 
$\rho_{0}(z=0)=0.336 \lambda, \rho_{0}(z=60 \lambda)=1.2 \lambda$ (the last row in Figure 2). Moreover, the uniformity of the longitudinal intensity can be varied by means of a Gaussian parameter $\sigma$ without the tube radius increasing.

\section{Conclusion}

The formation of thin light tube by tightly focused azimuthally polarized light beams was investigated in the paper.

It was theoretically shown that for the polar components the problem can be converted to a scalar one, and it can be solved by partitioning an aperture into narrow rings. It was shown in the analytical expression for a separate ring that due to the superposition of beams arriving to the focal region from different rings not only the full-aperture diffraction limit but also the limit corresponding to the Bessel function of the first order can be overcome. In this case, however, the intensity in the central part of the focal plane is significantly reduced, which practically leads to the tube rupture.

Various types of aperture apodization that allow forming of long thin light tubes were examined numerically. It was shown that using the diffraction axicon phase function slightly divergent light cone which is longer than $60 \lambda$ can be formed. The radius of the cone increases from $0.3 \lambda$ to $0.63 \lambda$. Light cone is displaced to the right or left from the focal plane depending on the axicon type (divergent or convergent).

To form the one-piece light tube that is symmetric with respect to the focal plane the Laguerre-Gaussian modes of higher order can be used. As the mode radial order decreases, the radius of the light tube and the energy falls. The divergence of this tube is in an agreement with the Gaussian beam and divergence in this case is more than that of axicon. In the center the radius was at the minimum $0.34 \lambda$ but at 60 wavelengths, it increased approximately from 4 -fold to $1.33 \lambda$. Because of the substantial divergence the tube intensity decreases rapidly when the distance from the focal plane grows. Longitudinal intensity of the tube can be made more uniform if the beam illuminating the lens is reduced in scale. Nevertheless the thickness of the formed tube increases.

To solve this problem it was proposed to use radial Airy-Gaussian functions. Divergence of the beam is almost the same but the uniformity of the longitudinal intensity can be varied by means of the Gaussian parameter without increasing the thickness of the tube.

\section{Acknowledgments}

The work was financially supported by the Russian Foundation for Basic Research (Grants 13-07-00266 and 13-0797004-r_povolzhe_a) and by the Ministry of Education and Science of Russian Federation (project 8231).

\section{References}

[1] S. Quabis, R. Dorn, M. Eberler, O. Glöckl, and G. Leuchs, "Focusing light to a tighter spot," Optics Communications, vol. 179, no. 1, pp. 1-7, 2000.

[2] R. Dorn, S. Quabis, and G. Leuchs, "Sharper focus for a radially polarized light beam," Physical Review Letters, vol. 91, no. 23, Article ID 233901, 2003.
[3] C. J. R. Sheppard and A. Choudhury, "Annular pupils, radial polarization, and superresolution," Applied Optics, vol. 43, no. 22, pp. 4322-4327, 2004.

[4] L. E. Helseth, "Mesoscopic orbitals in strongly focused light," Optics Communications, vol. 224, no. 4-6, pp. 255-261, 2003.

[5] C.-C. Sun and C.-K. Liu, "Ultrasmall focusing spot with a long depth of focus based on polarization and phase modulation," Optics Letters, vol. 28, no. 2, pp. 99-101, 2003.

[6] Y. Kozawa and S. Sato, "Sharper focal spot formed by higherorder radially polarized laser beams," Journal of the Optical Society of America A, vol. 24, no. 6, pp. 1793-1798, 2007.

[7] H. Wang, L. Shi, B. Lukyanchuk, C. Sheppard, and C. T. Chong, "Creation of a needle of longitudinally polarized light in vacuum using binary optics," Nature Photonics, vol. 2, no. 8 , pp. 501-505, 2008.

[8] S. N. Khonina and S. G. Volotovsky, "Controlling the contribution of the electric field components to the focus of a highaperture lens using binary phase structures," Journal of the Optical Society of America A, vol. 27, no. 10, pp. 2188-2197, 2010.

[9] S. N. Khonina, A. V. Ustinov, and E. A. Pelevina, "Analysis of wave aberration influence on reducing focal spot size in a highaperture focusing system," Journal of Optics, vol. 13, no. 9, Article ID 095702, 13 pages, 2011.

[10] G. T. di Francia, "Degrees of freedom of an image," Journal of the Optical Society of America, vol. 59, no. 7, pp. 799-804, 1969.

[11] F. M. Huang and N. I. Zheludev, "Super-resolution without evanescent waves," Nano Letters, vol. 9, no. 3, pp. 1249-1254, 2009.

[12] T. R. M. Sales and G. M. Morris, "Diffractive superresolution elements," Journal of the Optical Society of America A, vol. 14, no. 7, pp. 1637-1646, 1997.

[13] S. N. Khonina and I. Golub, "Enlightening darkness to diffraction limit and beyond: comparison and optimization of different polarizations for dark spot generation," The Journal of the Optical Society of America A, vol. 29, no. 7, pp. 1470-1474, 2012.

[14] S. N. Khonina and A. V. Ustinov, "Sharper focal spot for a radially polarized beam using ring aperture with phase jump," Journal of Engineering, vol. 2013, Article ID 512971, 8 pages, 2013.

[15] J. Bewersdorf, A. Egner, and S. W. Hell, “4pi-confocal microscopy is coming of age," Imaging \& Microscopy, vol. 4, pp. 24-25, 2004.

[16] L. E. Helseth, "Breaking the diffraction limit in nonlinear materials," Optics Communications, vol. 256, no. 4-6, pp. 435-438, 2005.

[17] N. Bokor and N. Davidson, "Tight parabolic dark spot with high numerical aperture focusing with a circular $\pi$ phase plate," Optics Communications, vol. 270, no. 2, pp. 145-150, 2007.

[18] H.-R. Noh and W. Jhe, "Atom optics with hollow optical systems," Physics Report, vol. 372, no. 3, pp. 269-317, 2002.

[19] V. A. Soifer, V. V. Kotlyar, and S. N. Khonina, "Optical microparticle manipulation: advances and new possibilities created by diffractive optics," Physics of Particles and Nuclei, vol. 35, no. 6, pp. 733-766, 2004.

[20] Y. Xue, C. Kuang, S. Li, Z. Gu, and X. Liu, "Sharper fluorescent super-resolution spot generated by azimuthally polarized beam in STED microscopy," Optics Express, vol. 20, no. 16, pp. 1765317666, 2012.

[21] S. N. Khonina and I. Golub, "How low can STED go? Comparison of different write-erase beam combinations for stimulated emission depletion microscopy," The Journal of the Optical Society of America A, vol. 29, no. 10, pp. 2242-2246, 2012. 
[22] B. Tian and J. Pu, "Tight focusing of a double-ring-shaped, azimuthally polarized beam," Optics Letters, vol. 36, no. 11, pp. 2014-2016, 2011.

[23] K. Lalithambigai, P. Suresh, V. Ravi et al., "Generation of sub wavelength super-long dark channel using high NA lens axicon," Optics Letters, vol. 37, no. 6, pp. 999-1001, 2012.

[24] W. Chen and Q. Zhan, "Three-dimensional focus shaping with cylindrical vector beams," Optics Communications, vol. 265, no. 2, pp. 411-417, 2006.

[25] X. Gao, J. Wang, H. Gu, and W. Xu, "Focusing properties of concentric piecewise cylindrical vector beam," Optik, vol. 118, no. 6, pp. 257-265, 2007.

[26] M. Abramowitz and I. A. Stegun, Handbook of Mathematical Functions, Courier Dover Publications, 1972.

[27] S. N. Khonina, N. L. Kazanskiǔ, A. V. Ustinov, and S. G. Volotovskiǔ, "The lensacon: nonparaxial effects," Journal of Optical Technology A, vol. 78, no. 11, pp. 724-729, 2011.

[28] S. N. Khonina, "Specular and vortical Airy beams," Optics Communications, vol. 284, no. 19, pp. 4263-4271, 2011. 

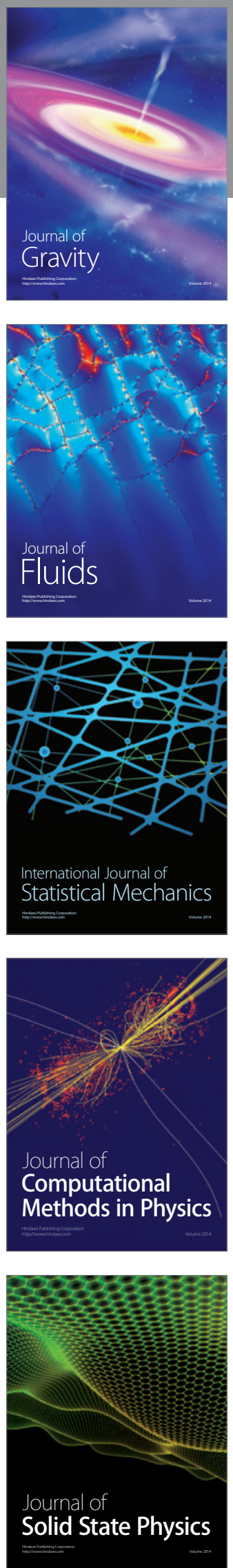

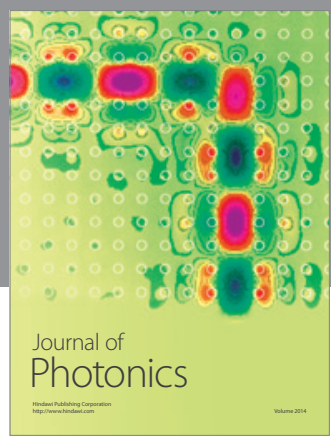

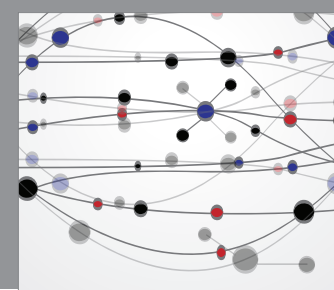

The Scientific World Journal

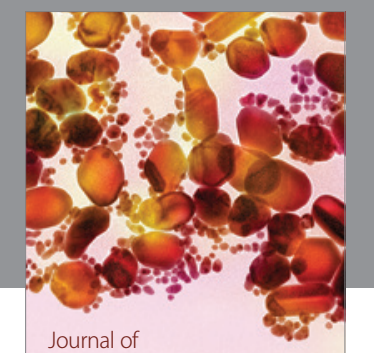

Soft Matter
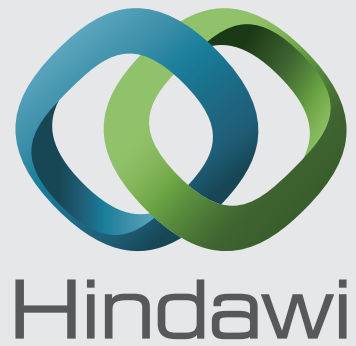

Submit your manuscripts at

http://www.hindawi.com
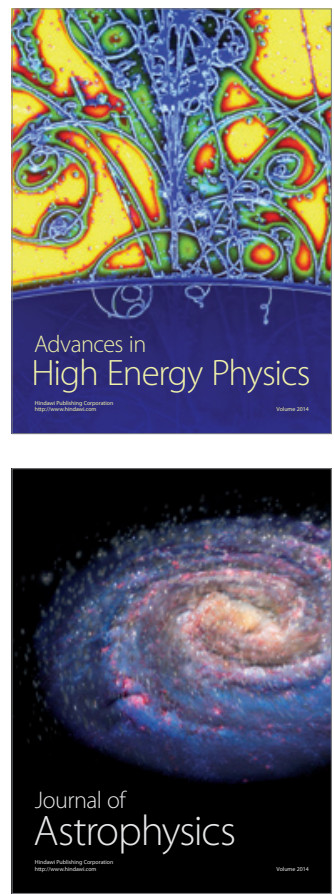
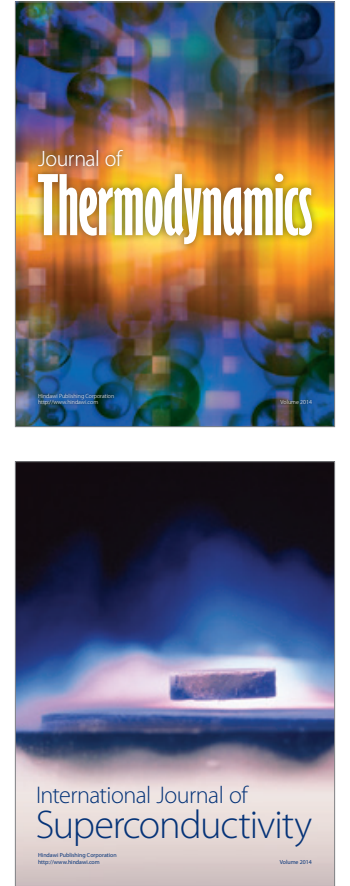
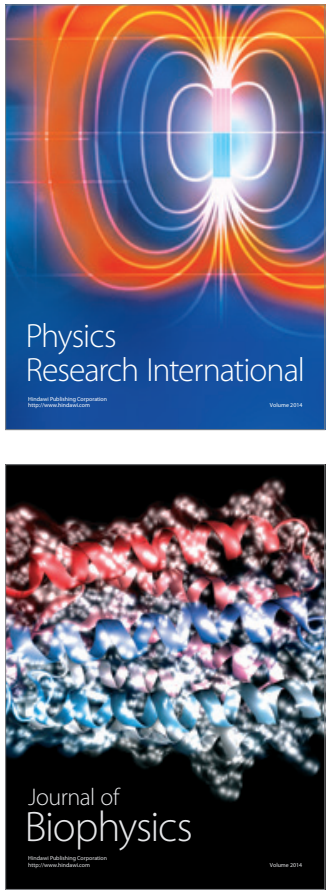
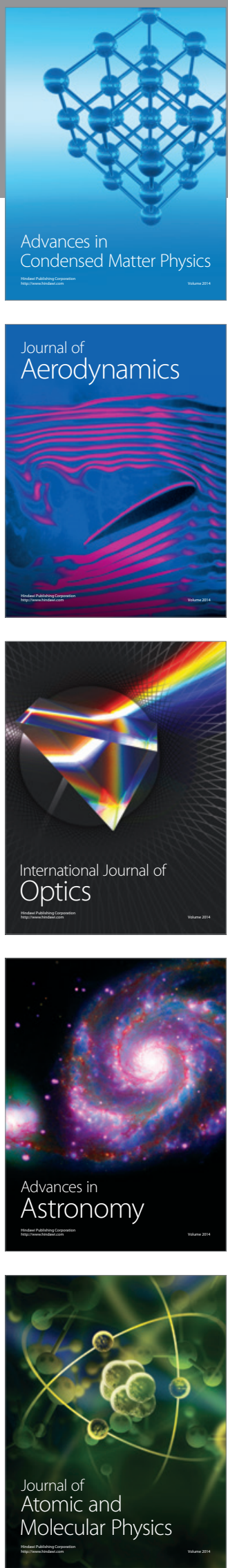